\title{
LA LIBERTAD DE EXPRESIÓN Y REGULACIÓN DE LA INFORMACIÓN EN ARGELIA
}

\author{
José Manuel Moreno Mercado \\ José Manuel Jiménez Cabello \\ Universidad de Granada
}

https://doi.org/10.33676/EMUI_nomads.55.19

Resumen: El presente artículo tiene como finalidad analizar la libertad de expresión y la regulación mediática de los medios informativos en Argelia durante el periodo 20072018. Desde las revueltas del año 2011, conocidas popularmente como la primavera árabe, todos los países del denominado mundo arabo-musulmán han experimentado cambios en sus marcos legislativos. Aunque dichas revueltas llegaron a derrocar los gobiernos de Túnez, Egipto o Libia, Argelia ha suscitado un gran interés por parte de los académicos al no producirse mutaciones significativas en la composición de su régimen. A través del análisis de diversas fuentes y escritos, el presente documento analiza los cambios en materia de libertad de expresión y regulación mediática en la última década.

Palabras clave: libertad de expresión, regulación mediática, Argelia

\section{Freedom of expression and regulation of information in Algeria}

Abstract: The present article takes aim at the analysis of freedom of speech and media regulation in Algeria from 2007 to 2018. Since the riots that took place in 2011, popularly known as the "Arab Spring", all the countries of the so called Arab-Muslim world have experienced changes in their legislative frames. Although the above mentioned riots went as far as to overthrow the governments of Tunisia, Egypt or Libya, Algeria has caused a great interest from academics, since it did not produce significative alterations in the composition of its regime. Through the analysis of diverse sources and documents, the present document analyzes the changes in topics such as freedom of speech and media regulation in the last decade.

Key words: freedom of speech, media regulation, Algeria

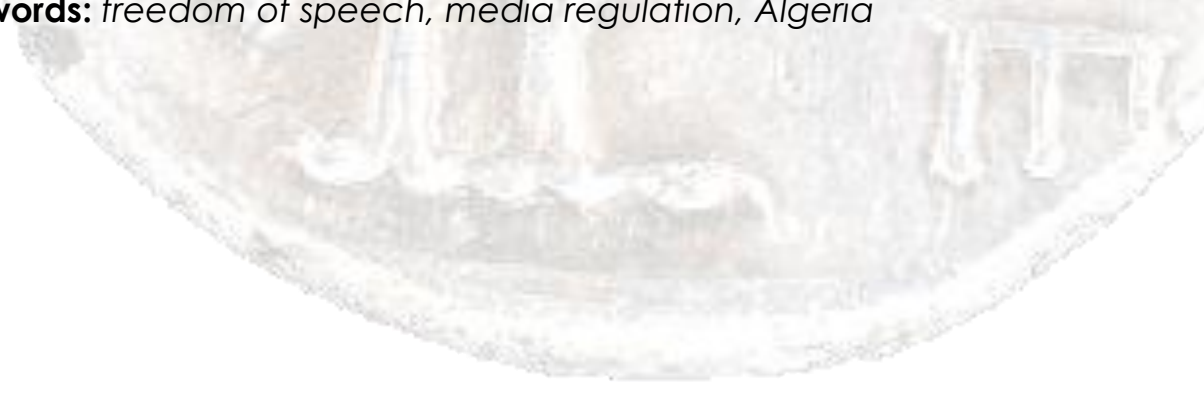

\section{Introducción}

Las revueltas populares de 2011 que se produjeron en numerosos países del denominado mundo árabe echaron abajo las tesis que sostenían que las sociedades árabes, y concretamente las magrebíes, no podían ser el epicentro de grandes movilizaciones y cambios sociales.

Tras la extensísima literatura producida a raíz de la Primavera Árabe o Despertar Árabe, pocos han sido los trabajos escritos en castellano que 
se han encargado de analizar y estudiar los cambios producidos en Argelia, un país vital para Europa, y sobre todo España, por su cercanía geográfica y especialmente por sus importantes recursos en hidrocarburos, concretamente gas.

Cuando nos aproximamos al estudio y análisis de los países que conforman el Magreb, encontramos un elemento en común que es la carencia de democracia, aunque Túnez ha dado grandes pasos en la consolidación de la democracia en los últimos años. Como señalan Carmelo Pérez Beltrán y Javier García-Marín (2015), "una de las tareas principales de la política comparada es la categorización de los diferentes regímenes políticos para, a través de las diversas taxonomías, explicar los componentes -variables- que constituyen la esencia de cada uno de ellos" (p. 70). Todas estas taxonomías se encuentran integradas a su vez en otros elementos como son las libertades públicas y los derechos civiles, que resultan cruciales para la elaboración de una Constitución normativa y la consolidación de un sistema democrático.

A pesar de que el concepto de "libertes públicas y derechos civiles" varía según el contexto histórico y la sociedad en la que nos encontremos, la inmensa mayoría de los autores afirman que podemos hablar de democracia cuando existe un sistema político que garantice una serie de derechos y libertades básicas, como son el derecho de una sociedad a elegir a sus representantes y el sometimiento institucional al imperio de la ley entre otros. Si atendemos a los principales autores comparativistas, observamos que existen tres tipologías de regímenes políticos: totalitarismos, autoritarismos y democracias, aunque estas tipologías pueden albergar otros elementos que los diferencien entre sí e incluso combinarse mutuamente, como es el caso de los regímenes híbridos (Szmolka, 2010).

La organización no gubernamental Freedom House y el medio de comunicación The Economist elaboran dos de los índices más reputados y citados por los autores que centran su análisis en las libertades públicas y civiles. En el caso del índice de Freedom House se registran la libertad de expresión, el sometimiento institucional al imperio de la ley y derechos individuales y colectivos, como la libertad de creencias y el derecho de asociación. Mientras que The Economist elabora su índice a partir de los resultados de 60 indicadores que se estructuran en cinco categorías diferentes: proceso electoral y pluralismo, libertades civiles, funcionamiento del Gobierno, participación política y cultura política. En cuanto a la clasificación que se realiza de los países seleccionados, ésta se realiza de la siguiente forma: países democráticos plenos (8-10 puntos), países democráticos defectuosos (6-7,9 puntos), países con regímenes híbridos (4-5,9 puntos) y países con regímenes autoritarios (puntación inferior a 4 puntos). 
Tabla 1. Índices de libertades civiles y de democracia en países seleccionados (2017-2018)*

\begin{tabular}{|l|l|l|}
\hline \multicolumn{1}{|c|}{ País } & \multicolumn{1}{|c|}{ Libertades Civiles (FH)** } & $\begin{array}{l}\text { Índice de Democracia } \\
\text { (EIU) }\end{array}$ \\
\hline Egipto & 6 (no libre) & 3.36 (régimen autoritario) \\
\hline Libia & 6.5 (no libre) & 2.32 (régimen autoritario) \\
\hline Marruecos & 5 (parcialmente libre) & 4.87 (régimen híbrido) \\
\hline Mauritania & 5.5 (no libre) & 3.82 (régimen autoritario) \\
\hline Siria & 7 (no libre) & 1.43 (régimen autoritario) \\
\hline Túnez & 2.5 (libre) & $\begin{array}{l}6.32 \\
\text { defectuosa) }\end{array}$ \\
\hline Argelia & 5.5 (no libre) & $\mathbf{3 . 5 6 \text { (régimen autoritario) }}$ \\
\hline Arabia Saudí & 7 (no libre) & 1.93 (régimen autoritario) \\
\hline Yemen & 6.5 (no libre) & 2.07 (régimen autoritario) \\
\hline Qatar & 5.5 (no libre) & 3.19 (régimen autoritario) \\
\hline Jordania & 5 (parcialmente libre) & 3.87 (régimen autoritario) \\
\hline
\end{tabular}

* Para FH, a mayor puntuación menor libertad. Para EIU, a mayor puntuación mayor libertad.

** Las categorías de "libre", "no libre" o "parcialmente libre" se conforman conjuntamente con las libertades políticas, no mostradas en la tabla, por lo que el mismo índice de libertades civiles puede mostrar una categoría diferente.

Fuente: Freedom House (FH), 2018; The Economist Intelligence Unit (ElU), 2017.

Desde el año 2007, el autoritarismo en Argelia se ha mantenido de forma más o menos constante, aunque los índices de democracia mejoraron levemente durante el periodo post-primavera árabe. No obstante, diversas organizaciones no gubernamentales y fundaciones han alertado sobre la preocupante situación en materia de libertad de expresión y prensa, ante la prohibición de sitios webs, presiones sobre medios de comunicación y autocensura.

El autoritarismo en Argelia dio un paso más cuando en enero del año 2016 las autoridades disolvieron el Departamento de Información y Seguridad, sustituyéndolo por un nuevo servicio de inteligencia, Dirección de Servicios de Seguridad, que pasaba a depender directamente del presidente (Iriarte, 2016).

Según Reporteros Sin Fronteras, Argelia se sitúa en la posición 136 del ranking, junto con países como Marruecos, Myammar, India y Palestina, 
calificando la situación de libertad de prensa como muy deteriorada (2018). Durante el año 2016 las autoridades argelinas encarcelaron a cuatro blogeros y multaron a la activista Zoulikha Belarbi por difamaciones y ofensas a la figura del presidente Bouteflika. Por otro lado, a finales del pasado año el periodista Mohammed Tamalt falleció en prisión tras una larga huelga de hambre, siendo el primer periodista muerto en prisión por expresar sus opiniones en Facebook. Al igual que los anteriores, el periodista británico-argelino se encontraba encarcelado por expresar opiniones críticas a la figura del presidente, acusado de "insultar a un cuerpo constituido" y "atentar contra la figura del Presidente", tipos delictivos recogidos en el Código Penal.

Para analizar la evolución en Argelia de la libertad de prensa y expresión derechos fundamentales de gran importancia ya que sin estos no pueden constituirse los demás, el siguiente estudio se organiza de la siguiente manera. En primer lugar, se realizará un análisis explicativo sobre los sucesos que afectaron a Argelia durante las revueltas del 2011 para contextualizar su situación. En segundo lugar, se analiza el marco regulatorio mediático y su evolución desde el año 2007, así como un estado de la cuestión sobre la libertad de expresión y la libertad de prensa, utilizando distintas fuentes de carácter cuantitativo y cualitativo.. Por último, se proponen unas conclusiones analíticas de la situación actual.

\section{Las revueltas de 2011 en Argelia}

Aunque el término primavera árabe no es nuevo, ya se acuñó la expresión "primavera" en las revueltas que se produjeron en los años ochenta en Argelia, mediáticamente se ha relacionado con las revueltas de 2011 que tuvieron su origen en Túnez y se fueron expandiendo progresivamente al resto de países del mundo arabomusulmán.

Este fenómeno, que ha sacudido a las sociedades árabes, y especialmente las magrebíes, ya que los tres líderes que fueron derrocados pertenecían a países de África del Norte (Libia, Túnez y Egipto), ha afectado en mayor o menor medida a todos aquellos regímenes autoritarios tan prolongados en el tiempo que parecían inmutables. Hecho fácilmente visible en los procesos reformistas vividos en países como Marruecos o Jordania, y mucho más evidente en los conflictos que se han desatado en Yemen y en la República Árabe Siria, cobrándose este último casi 470.000 víctimas mortales (Boghani, 2016).

Pese a que las revueltas y las protestas de carácter violento, especialmente por el desempleo y la pobreza, han estado presentes en el panorama social argelino desde antes de las revueltas de 2011, el caso de Argelia ha sido especialmente llamativo por las escasas consecuencias para su sistema político y su establishment. Aunque 
diversos autores mantienen que el malestar político y social en la sociedad civil argelina ha sido similar al de sus vecinos magrebíes, las cruentas consecuencias de la guerra civil (1991-2002), así como unos eficientes mecanismos inhibidores gubernamentales, han sido señaladas como elementos clave de la actual situación (Thieux, 2012).

A principios del mes de enero de 2011, el precio de los víveres, especialmente aceite de cocina y azúcar, experimentó una inflación del $30 \%$, lo que produjo un profundo malestar social entre la población que se materializó en varias protestas en distintos puntos del país. Coincidiendo con las revueltas en la vecina Túnez, que más tarde desembocarían en el derrocamiento de Zine El Abidine Ben Ali, las revueltas iniciadas en Argel se extendieron a otras wilayas donde los manifestantes prendieron fuego a varios edificios públicos produciendo numerosos disturbios y enfrentamientos con las fuerzas de seguridad.

Tras el recorte de un $41 \%$ a las tasas sobre el azúcar y el aceite, el número de protestas disminuyó, hecho que dejó claro que, como afirman Ahmed Aghrout y Lorena Murillo, "los primeros motines de enero tenían más que ver con las privaciones socioeconómicas que con una lista bien articulada de demandas políticas" (2012: 7). Sin embargo, tras la caída de Ben Ali y, su posterior exilio hacia Arabia Saudita, ciertos grupos políticos opositores, como la Agrupación por la Cultura y la Democracia (RCD), crearon la denominada Coordinación Nacional por el Cambio y la Democracia (CDND) con la intención de imitar el proceso experimentado en Túnez. Aunque la CDND amenazó en reiteradas ocasiones con la convocación de huelgas masivas, las divisiones internas entre la gran amalgama de componentes y la falta de coordinación entre otros grupos opositores hicieron que el proceso revolucionario en Argelia fracasara, en comparación con otros movimientos árabes de la región.

Como señalan la mayoría de los estudiosos, la razón de este fracaso revolucionario parece ser la distancia entre las demandas socioeconómicas y las políticas, puesto que se siguieron convocando huelgas y protestas de otros colectivos de carácter socioeconómico (como estudiantes, académicos, desempleados...), no conexionados con la CDND.

No obstante, las revueltas tuvieron su impacto, el régimen de Bouteflika anunció la supresión del estado de emergencia vigente en el país desde 1992, que prohibía las manifestaciones, hecho denunciado sistemáticamente por la oposición ya que los índices de violencia habían descendido considerablemente. Después del levantamiento del estado de emergencia, Bouteflika anunció la promesa de revisar la Constitución para traer al país una democracia más representativa mediante la reforma de la legislación correspondiente a elecciones, representación institucional, asociaciones y medios de comunicación. 
Resulta vital remarcar que durante la supresión del estado de emergencia, el régimen articuló una nueva ley que permitía a las fuerzas de seguridad mantener las mismas funciones que durante la vigencia del estado de emergencia, aunque, por supuesto, no fue publicitada por el ejecutivo.

Con un apoyo menor en el legislativo, por las diversas coaliciones islamistas en el Parlamento y un, cada vez más, reducido apoyo social, el gobierno de Bouteflika proclamó la creación de la Comisión Nacional de Consultas sobre las Reformas Políticas (CNCRP), para así recoger todas las demandas de los diferentes grupos políticos relativas a la reforma constitucional y legislativa. Numerosas formaciones y asociaciones políticas incidieron en la necesidad de reformar la Constitución para así dar base a un sistema más democrático y pluralista, aunque varias personalidades políticas influyentes, como Hocine Ait Ahmed o Mokdad Sifi, decidieron no participar en el periodo de consultas llegando en algunos casos, como el de Ait Ahmed, a catalogar el proceso como un "circo político" (Aghrout y Murillo, 2012).

Pese al acuerdo para la aprobación de enmiendas a la Constitución y a la legislación referida al sistema electoral y al derecho de asociación por el Parlamento, el presidente Bouteflika, enfermo y cada vez más alejado de la vida pública, volvió a ganar las elecciones presidenciales de 2014, bajo un clima de tensión política donde prácticamente toda la oposición denunció un masivo fraude electoral.

\section{Libertad de expresión y medios de comunicación (2007-2018)}

Tomando como referencia a Inmaculada Szmolka, los derechos y las libertades públicas pueden categorizarse en tres estados: estar plenamente garantizados, ser restrictivos, o ser muy restrictivos (2010).

El presente estudio se centra en el análisis de la libertad de expresión, fuertemente ligada al derecho de libertad de prensa, columna vertebral de donde emanan el resto de libertades públicas.

Para analizar la evolución de dichas libertades en Argelia, y realizar un estado de la cuestión actual, se han usado diversas fuentes e índices que ofrecen una gran cantidad de información, tanto a nivel cuantitativo como cualitativo:

1) Se han utilizado distintos índices elaborados por organizaciones internacionales, de gran y reconocido prestigio en el mundo académico, especializadas en los temas de democracia, democratización y cambio político (BTI, Freedom House Reports, y los informes de Reporteros Sin Fronteras) 
2) Distintos textos legislativos y disposiciones legales interesantes para este texto, como son la Constitución, el Código Penal o la Ley de Asociación.

3) Datos del Observatorio sobre la actividad yihadista en el Magreb y el Sahel Occidental del Grupo de Estudios en Seguridad Internacional (GESI) para conocer cómo ha sido la cobertura del terrorismo en Argelia.

El periodo de análisis seleccionado, se centra en los últimos once años (periodo 2007-2018). La selección de los años se ha elaborado con la intención de explorar la evolución de los derechos anteriormente citados en el periodo pre y post-primavera árabe.

La Tabla 1, expuesta en la introducción, muestra los índices de democracia relativos a países del mundo árabe seleccionados durante el período 2017-2018. El elemento común, señalado anteriormente, es la falta de democracia, aunque los efectos de la primavera árabe han afectado en mayor o menor medida en todos los países que han experimentado protestas y revueltas sociales. Tras la caída de Ben Ali y Muammar el Gaddafi en las vecinas Túnez y Libia, el ejecutivo de Abdelaziz Bouteflika anunció el fin del estado de emergencia instaurado desde el golpe de Estado de 1992 que prohibía las concentraciones y manifestaciones por motivos de seguridad nacional. En febrero de 2016 el Parlamento argelino aprobó una serie de reformas constitucionales prometidas por Bouteflika, que tendrían como fin la apertura y democratización del régimen tras más de 24 años de autoritarismo.

Aunque los efectos de las revueltas de 2011 hacen suponer que los sistemas autoritarios no son sostenibles a largo plazo, y por consiguiente, son sólo una fase transicional hacia la democracia (Pace y Cavatorta. 2012), el efecto democratizador de la primavera árabe, que se presuponía en un primer momento, no se ha producido, sino que los regímenes han realizado reformas (como el caso de Marruecos), virando hacia regímenes híbridos. Incluso algunos autores, como Alfred Stepan y Juan Linz (2013), han creado una nueva tipología Hamada sistema híbrido autoritario-democrático para explicar los cambios de rumbo de algunos regímenes del mundo árabe. Un ejemplo sería el caso de Egipto donde, a pesar de sufrir importantes revueltas sociales y políticas, no se ha logrado implementar un sistema democrático, sino que han aparecido nuevos modelos híbridos que han vuelto a evolucionar hacia sistemas autoritarios.

A pesar de los esfuerzos de Bouteflika por pacificar el país (véase el caso de la Ley de Amnistía) y mostrar una imagen "democratizadora del régimen", sus victorias electorales (siempre bastante amplias) han estado salpicadas por la polémica. El artículo 85 de la Constitución establece que "el Presidente de la República es elegido por sufragio universal, directo y secreto", pero el posicionamiento de la élite militar es un elemento vital para la configuración del poder ejecutivo en Argelia, 
hecho denunciado sistemáticamente por la oposición como un fraude electoral y un ataque a las libertades públicas.

Por lo tanto, Argelia puede ser encasillada dentro del denominado autoritarismo competitivo debido a que, aunque la Constitución argelina de 1996, modificada en 2016, configura un sistema democrático basado en el pluralismo político, elecciones libres y reconocimiento de las libertades públicas, las decisiones institucionales provienen de ciertos lobbies (especialmente el militar) y centros de poder fuera del marco constitucional (Levitsky y Way, 2004). Otro aspecto destacable de este autoritarismo, es la exclusión de ciertos grupos, con amplia representatividad, como es el caso de los islamistas y algunos movimientos bereberes ubicados en el norte del país, que son vistos por el régimen como una amenaza a su supervivencia.

Tabla 2. Valoraciones sobre la calidad de la democracia o sobre el estado de las libertades fundamentales en Argelia por varias organizaciones

\begin{tabular}{|c|c|c|c|c|c|c|c|c|c|c|c|c|}
\hline & $\begin{array}{c}200 \\
7\end{array}$ & $\begin{array}{c}200 \\
8\end{array}$ & $\begin{array}{c}200 \\
9\end{array}$ & $\begin{array}{c}201 \\
0\end{array}$ & $\begin{array}{c}201 \\
1\end{array}$ & $\begin{array}{c}201 \\
2\end{array}$ & $\begin{array}{c}201 \\
3\end{array}$ & $\begin{array}{c}201 \\
4\end{array}$ & $\begin{array}{c}201 \\
5\end{array}$ & $\begin{array}{c}201 \\
6\end{array}$ & $\begin{array}{c}201 \\
7\end{array}$ & $\begin{array}{c}201 \\
8\end{array}$ \\
\hline RSF* & $\begin{array}{l}40.5 \\
0\end{array}$ & $\begin{array}{l}31.3 \\
3\end{array}$ & $\begin{array}{l}49.5 \\
6\end{array}$ & $\begin{array}{l}47.3 \\
3\end{array}$ & $\begin{array}{l}56.0 \\
0\end{array}$ & & $\begin{array}{l}36.5 \\
4\end{array}$ & $\begin{array}{l}36.3 \\
6\end{array}$ & $\begin{array}{l}42.8 \\
3\end{array}$ & $\begin{array}{l}41.6 \\
9\end{array}$ & $\begin{array}{l}42.8 \\
3\end{array}$ & $\begin{array}{c}43.1 \\
3\end{array}$ \\
\hline$\left.\mathrm{BT}\right|^{* *}$ & & 4.72 & & 4.86 & & 4.78 & $\mathrm{~N}$ & 5.11 & & 5.11 & & 4.98 \\
\hline $\begin{array}{l}\mathrm{FH}^{* *} \\
*\end{array}$ & 5.5 & 5.5 & 5.5 & 5.5 & 5.5 & 5.5 & 5.5 & 5.5 & 5.5 & 5.5 & 5.5 & 5.5 \\
\hline
\end{tabular}

* RSF a menor número mayor libertad de prensa

** BTI: 1-10, a mayor número mayor calidad democrática

*** FH: 1-7 a menor número mayor democracia

Fuente: Reporteros sin Fronteras (RSF); Freedom House (FH); BTI.

Por otro lado, como se puede observar en la Tabla 2, la libertad de expresión y de prensa en Argelia, así como la calidad de la democracia, han sufrido pequeñas fluctuaciones que no han otorgado mejoras significativas desde las revueltas populares del año 2011, aunque se experimenta un sensible retroceso en los últimos años. En la tabla se exponen los datos de Reporteros Sin Fronteras que realizan un análisis anual sobre la libertad de prensa a partir de un cuestionario de 43 ítems, ampliamente valorado en el ámbito científico. En lo relativo al país magrebí, el informe de RSF denuncia desde hace años severas restricciones de libertad de expresión y prensa, lo que ha llevado en mayo de 2018 al gobierno argelino a etiquitar el informe como fake news. 
Para conocer y analizar el status del sector de la información y la comunicación pública, es necesario revisar una serie de textos que garanticen, en primer lugar, las libertades fundamentales, incluidas la libertad de opinión, de expresión, prensa e información (Durán Cenit y García Marín, 2015: 198). Dichos textos suelen incluir la Constitución como norma fundamental del Estado, y la legislación relativa a la definición de los derechos fundamentales, especialmente la relativa al derecho de acceso a la información y la libertad de prensa.

En el caso de Argelia nos encontramos con una Carta Magna vagamente definida con respecto a la libertad de expresión. Son los artículos 42 (libertad de consciencia y pensamiento), 44 (diseminación de las opiniones y la creatividad intelectual), 48 (libertad de expresión y asociación), 50 (libertad de prensa), 51 (libertad de información) y 54 (derecho a crear asociaciones), los que hacen mención a ella. Especialmente interesante nos resulta el contenido expuesto en el artículo 50 y 51 para nuestro propósito, debido a que en ambos se encuentran elementos cercenadores del propio derecho reconocido:

"La libertad de prensa escrita y audiovisual expresada a través de los medios de comunicación queda garantizada y no puede ser restringida por ninguna forma de control previo. Esta libertad no puede usarse para perjudicar a la dignidad de otros ciudadanos, los derechos y las libertades. La publicación sin restricciones de información, ideas, imágenes y opiniones está garantizada por el marco legal bajo los principios de respeto a los valores éticos, culturales, religiosos y principios de la nación. Las ofensas de prensa pueden no estar sujetas a castigos de privación de libertad".

En esta línea, el art. 51 también establece limitaciones al derecho a la información:

"La adquisición y transferencia de información, documentos y estadísticas queda garantizada a los ciudadanos. El ejercicio de este derecho no puede perjudicar la vida privada de los demás, sus derechos, sus intereses legales contractuales y los requerimientos relativos a la seguridad nacional. La ley establecerá modalidades de ejercicio para este derecho".

Como se puede observar legalmente, existe un reconocimiento constitucional de los derechos y libertades públicas al igual que en la mayoría de cartas magnas del resto del mundo. No obstante, ciertas limitaciones como las expuestas pueden materializarse a partir de decretos ejecutivos y procedimientos legislativos ordinarios que han dado lugar un enorme volumen de legislación, de amplia y ambigua interpretación, que suponen un impedimento a la práctica de dichos derechos. Un ejemplo claro lo encontramos en el procesamiento del periodista Saïd Chitour en 2017, acusado de proporcionar información a un país extranjero, delito tipificado en el artículo 65 del Código Penal que contempla penas que incluyen la cadena perpetua. La práctica 
de jueces y fiscales, a la hora de interpretar los artículos del Código Penal, ha sido ampliamente catalogada como restrictiva, especialmente en la aplicación de los artículos recogidos en las secciones II y III, relativas a atentados contra la defensa y la economía nacional y atentados y complots contra la autoridad del Estado y la integridad territorial respectivamente.

Como se puede observar, los regímenes autoritarios presentes en la región MENA poseen Constituciones que garantizan los derechos y libertades públicas, pero en algunos casos, como el argelino, ya se vislumbran ciertos mecanismos limitadores (Marhuenda García, 2012). Por supuesto, la oleada de protestas comenzadas en el año 2011 ha provocado que el ejecutivo argelino haya optado por reformar ampliamente sus textos legales catalogadas como "reformas topdowns estrechamente controladas" (Driss-Ait Hamadouche, 2012). A pesar de las novedosas reformas en materia de asociación, como la desaparición de dar publicidad en un periódico de tirada nacional a una nueva asociación (art. 50 de Ley N. ${ }^{\circ} 12-06$ del 12 de enero de 2012, referente a la asociaciones), el enorme y ambiguo volumen de elementos y prerrogativas legales introducidos se han traducido en nuevos mecanismos de control mucho más estrictos (Pérez Beltrán, 2014: 193).

Ya sea en el Código Penal, en la Constitución, en la Ley de Asociación o en la Ley Antiterrorista, donde únicamente Argelia y Túnez poseen una legislación específica contra el terrorismo por sus problemas históricos con el Islam radical (Tamburini, 2018: 4), Argelia es un país que ha producido modificaciones sustanciales en su legislación. No obstante, se podría afirmar que el balance ha resultado negativo porque, ya que a pesar de los avances aperturistas, éstos no han sido suficientes para desclasificar a Argelia como país autoritario, con una situación comparable a países tan restrictivos como Turquía o Mauritania. 
Gráfico 1. Cobertura del terrorismo por la prensa argelina (2017)

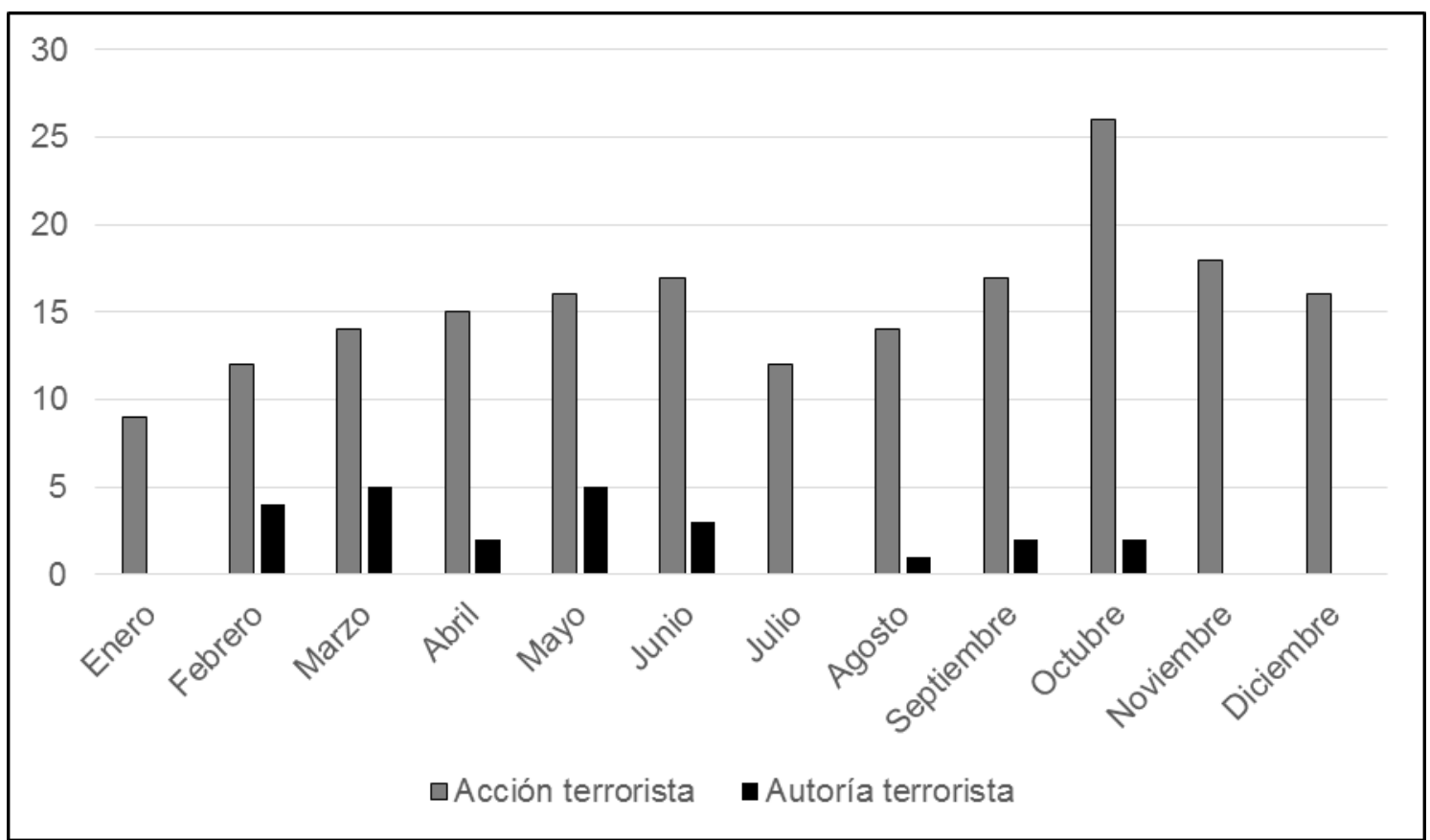

Fuente: elaboración propia a partir de los datos del Observatorio sobre la actividad yihadista en el Magreb y el Sahel Occidental (2017).

El pluralismo político, y por tanto, la libertad de expresión, donde radican el conflicto y el debate de ideas, constituyen la columna vertebral de cualquier democracia (Schattschneider, 1975), incluyendo, por supuesto, los medios de comunicación social. No obstante, como señalan diversos autores, los medios de comunicación han sido obviados de los manuales de política comparada, al considerarse plenamente dependientes de la situación política general no se ha tenido en cuenta su rol como elementos socializadores (García-Marín, 2013: 161). Sin embargo, dentro del campo de la comunicación política las teorías de encuadres (frames), así como diversos elementos procedentes de la psicología cognitiva, como el priming, indican que los arquetipos clásicos de la propaganda, con audiencias pasivas, han quedado histórica y académicamente obsoletos. De ahí que sucesos como la Primavera Árabe se hayan convertido en uno de los más impredecibles de los últimos tiempos, incidiendo numerosos autores en el importante papel de las herramientas sociales de comunicación (Seo y Ebrahim, 2016: 232).

Aunque la comunicación pública relativa al caso del terrorismo constituye un sector problemático, en el que se articulan las estructuras de seguridad interior con los niveles políticos (Jaime Jiménez, 2017: 76), el gráfico 1 nos otorga un buen retrato de la situación actual en Argelia. Los datos extraídos del Observatorio sobre la actividad yihadista en el 
Magreb y el Sahel Occidental' muestran una tendencia a obviar el reconocimiento de la autoría de acciones terroristas en el país. Empero, si se analizan de una manera más amplia encontramos aspectos significativos, como una elevada cobertura de acciones militares contraterroristas, vaga definición de las acciones de los actores terroristas, cierta tendencia a identificar a elementos subversivos a grupos ya disueltos como el $\mathrm{GIA}^{2}$ o ausencia de crítica a la acción gubernamental. Este último elemento se encuentra estrechamente relacionado con el uso de la difamación como herramienta de autocensura, ampliamente utilizada en regímenes autoritarios. Un ejemplo claro lo encontramos en lo expuesto en el informe del International Press Institute (2009): "Difamar o insultar a altos cargos continua acarreando condenas de prisión en muchos países del área MENA. En Argelia, la difamación de altos cargos y órganos del Estado han estado criminalizadas desde 2001 y al menos hasta febrero de 2006 es ilegal criticar las acciones de las fuerzas de seguridad acaecidas durante los años noventa".

A pesar de ello, Argelia ha sido el país que más fluctuaciones legales y políticas ha ido experimentando dentro de los países que conforman el Magreb (García-Marín, 2017: 240). En el año 2012 se abolieron las penas de prisión relativas a ofensas por parte de la prensa escrita, reguladas en la Ley de Prensa, y un año más tarde el ejecutivo de Bouteflika permitió la entrada de medios de televisión privados, rompiendo con el monopolio estatal relativo a la televisión, y creando la Autoridad para la Regulación Audiovisual (ARAV). El monopolio informativo materializado hasta entonces por la Radio Télévision Algérienne (RTA) basaba su existencia en disposiciones legales, como el artículo 5 del Código de Información de 1982, que estipulaba "los directores [de medios de comunicación] tendrán la autoridad exclusiva para implementar las instrucciones del poder político" (Mostefavi, 2013: 167), mantenida posteriormente por el Acta de Información de 1990. Sin embargo, los controles sobre la comunicación pública siguen siendo notablemente restrictivos sobre ciertos temas, como la salud del presidente o la presencia de Daesh en el país, llegando a cerrar cadenas escritas y audiovisuales, como en los casos de Atlas TV y El Watan (Audije, 2016).

\section{Conclusiones}

A lo largo de esta reflexión creemos haber mostrado suficientemente la situación actual en materia de libertad de expresión y prensa en Argelia y su comparativa histórica. Así, dicho texto es consciente de las múltiples

\footnotetext{
1 Utilizando fuentes abiertas, principalmente medios nacionales de la prensa argelina como Algérie Presse Service (APS) o Tout sur l'Algérie (TSA) entre otros.

2 Siglas de Grupo Islámico Armado.
} 
incógnitas que este deja en el aire y que, por otro lado, puedan ser una correcta guía de inicio para investigaciones futuras. No olvidamos señalar que la multitud de disposiciones y textos legales hacen inviable realizar un análisis completo de la situación. Aun así, consideramos que las fuentes utilizadas aportan datos suficientemente relevantes sobre la actual situación socio-política en el país magrebí.

Por supuesto, es indiscutible afirmar que las revueltas populares de la región MENA del año 2011 han afectado al sistema político, pero a su vez sería descabellado decir que se han producido cambios reales relevantes. Todo esto nos lleva a considerar a Argelia como un sistema político de carácter autoritario con derechos y libertades públicas reconocidas, pero con un funcionamiento claramente restrictivo. Es cierto que se han producido reformas significativas, como el fin del monopolio estatal de los medios de comunicación, pero ninguno de los índices reputados consultados, ni la bibliografía especializada en el tema en cuestión, señalan fluctuaciones positivas considerables. Esto sucede ya que las reformas emprendidas por el gobierno no se ven realmente reflejadas en la práctica. Encontramos un ejemplo en la escasa información que se da por parte de los medios sobre cuestiones como el terrorismo.

A nuestro parecer existen dos cuestiones fundamentales a la hora de aproximarse al estudio de la realidad argelina en los próximos años. En primer lugar, las reformas políticas que puedan producirse en el país tras la muerte de Abdelaziz Bouteflika. Si bien es cierto que el presidente, debido a su delicado estado de salud, no ejerce poderes políticos reales, la tentación de imponer un candidato por parte del estamento militar siembra ciertas dudas en cuanto al futuro político del país (Alvarado, 2013). Por otro lado, creemos que sería interesante estudiar los efectos de las reformas que están acaeciendo en Estados cercanos, con peso político relevante en la región, como son Túnez y Marruecos.

En definitiva, podríamos asegurar que la realidad presente muestra cierto equilibrio entre el mantenimiento del status-quo y la derogación de diversos elementos restrictivos (Mostefaoui, 2013). Por supuesto, temas como el tratamiento del terrorismo, la libertad de las columnas de opinión y, especialmente, aunque no se ha incluido en este documento, el uso de Internet, serán variables a tener en cuenta para investigaciones futuras.

\section{Referencias bibliográficas}

Aghrout, A. \& Murillo, L. (2012). La "Primavera Árabe" de Argelia: ¿̇Tanto para nada?. Foro Internacional, 412-433.

Alvarado, D. (2013). Argelia en transición. Después de Bouteflika ¿quién?. Opinión CIDOB. 
Audije, P. (2016). Argelia: libertad de prensa y fin de reinado. Periodistas. Descargado de https://periodistas-es.com/argelia-libertad-prensafin-reinado-73226

BTI. (2018). Governance in International Comparison. Transformation Index BTI. Descargado de https://www.bertelsmannstiftung.de/en/publications/publication/did/transformation-indexbti-2018-1/

Driss-Ait Hamadouche, L. (2012). L'Algérie face au printemps arabe: pressions diffuses et résilence entretenue. Annuaire IEMED de la Méditerranée.

Dúran Cenit, M \& García-Marín, J. (2015). Libertad de expresión y regulación mediática en la Turquía de Erdogan. En I. Álvarez-Ossorio (Ed.), La Primavera Árabe revisitada (pp. 191-210). Pamplona: Aranzadi.

EIU. (2017). The Economist Intelligence Unit's Democracy Index. Descargado https://infographics.economist.com/2018/Democracylndex/ de Freedom House (2018). Freedom in the World 2018: Democracy in Crisis. Freedom House. Descargado de https://freedomhouse.org/sites/default/files/FH_FITW_Report_2018_Final_S ingl ePage.pdf

García-Marín, J. (2013). Panorama de los medios de comunicación en Oriente Medio. En I. Álvarez-Ossorio (Ed.), Sociedad civil y contestación en Oriente Medio y Norte de África (pp. 159-182). Barcelona: CIDOB.

García-Marín, J. (2017). Media and media freedom. En I. Szmolka (Ed.), Political change in the Middle East and North Africa after the Arab Spring (pp. 231-256). Edimburgo: Edinburhg University Press.

International Press Institute (2009). Middle East and North Africa Institute.

Iriarte, D. (2016).La disolución del servicio secreto trata de consolidar el poder de Bouteflika. El Confidencial. Descargado de https://www.elconfidencial.com/mundo/2016-01-29/lucha-de-poder-enargelia- bouteflika-ha-vencido-al-servicio-de-inteligencia_1142953/

Jaime Jiménez, O. (2017). Comunicación en seguridad pública: la interacción entre políticos, policías, medios de comunicación y sociedad. En R. Martínez (Ed.), Comunicación Política en Seguridad y Defensa (pp. 63-78). Barcelona: CIDOB.

Levitsky, S \& Way, L. (2004). Elecciones sin democracia. El surgimiento del autoritarismo competitivo. Estudios Políticos, 24, 159-176.

Marhuenda García, F. (2012). La libertad de expresión y la ciberrevolución en el mundo árabe. Revista general de derecho público comparado, 11. 
Mostefaoui, B. (2013). Algerian public authorities in the fase of transnational media competition: between status quo and deregulation. En T. Guaaybess (Ed.), National broadcasting and state policy in Arab countries (pp. 166-187). Londres: Palgrave Macmillan.

Pace, M \& Cavatorta, F. (2012). The Arab uprisings in theoretical perspective-an introduction. Mediterranean Politics, 17, 2, 125-138.

Pérez Beltrán, C. (2014). Reformas jurídicas en Argelia tras la "primavera árabe": la nueva "Ley de Asociación de 2012". Anaquel de estudios árabes, 25, 177-194.

Pérez Beltrán, C \& García-Marín, J. (2015). Libertades públicas en Túnez tras las revueltas de 2011. Revista CIDOB d'Affers Internationals, 109, 69-90.

Rodríguez, B. (2017). Observatorio sobre la actividad yihadista en el Magreb y el Sahel Occidental. Grupo de Estudios en Seguridad Internacional. Descargado de http://www.seguridadinternacional.es/?q=es/content/observatoriosobre-la- actividad-yihadista-en-el-magreb-y-el-sahel-occidental RSF. (2018). Clasificación Mundial 2018. Reporteros sin Fronteras. Descargado de https://www.rsf-es.org/grandes-citas/clasificacion-porpaises/

Schattschneider, E. (1975). The Semi-Soverign People: A Realist's View of Democracy in America. Belmont: Wadworth Publishing.

Seo, H \& Ebrahim, H. (2016). Visual propaganda on Facebook: A comparative analysis of Syrian conflicts. Media, War \& Conflicts, 9, 3, 227-251.

Stepan, A \& Linz, J. (2013). Democratization Theory and the Arab Spring. Journal of Democracy, 24, 2, 15-30.

Szmolka, I. (2010). Los regímenes políticos híbridos: democracias y autoritarismos con adjetivos, su conceptualización, categorización y operacionalización dentro de una tipología de regímenes políticos. Revista de Estudios Políticos, 147, 103- 135.

Tamburini, F. (2018). Anti-Terrorism Laws in the Maghreb Countries: The Mirror of a Democratic Transition that Never Was. Journal of Asian and Africain Studies, 1-16.

Thieux, L. (2012). El papel de la sociedad civil argelina en las perspectivas de cambio en Argelia. Revista general de derecho público comparado, 11. 